\title{
Indoor Air Pollution and Low Birth Weight- A Cross-Sectional Study
}

\author{
Devkota $\mathrm{N}^{1}$, Kodkany BS ${ }^{2}$, Angolkar $\mathrm{M}^{3}$, Pokhrel S ${ }^{4}$ \\ ${ }^{I}$ (Department of Public Health, KLE University, India) \\ ${ }^{2}$ (KLE Research Foundation, KLE University, India), \\ ${ }^{3}$ (Department of Public Health, KLE University, India) \\ ${ }^{4}$ (Department of Public Health, KLE University, India)
}

\begin{abstract}
Smokes from solid fuels used in developing countries contain combustion pollutants whose exposure is expected to have an impact on adverse pregnancy outcomes (low birth weight, still births). The association between maternal exposures to household air pollution (HAP) and low birth weight have been described in number of studies; however only few studies have been conducted in India to establish this association. Thus, our objective was to identify the association between exposure of pregnant women to indoor air pollution and birth weight of a newborn. A community based cross-sectional study was conducted in Machche Village of Kinaye primary health center for a period of one and half year. The data were collected from the pregnant mothers attending the antenatal checkup visits to the sub-centers during the study period. Universal Coverage was followed in this study. i.e. 254(All registered pregnant mothers during January to November 2012). The prevalence of low birth weight was found to be $2.8 \%$. The study didn't find any significant association between types of fuel used, exposure of the mothers to passive smoke and birth weight of a newborn. This resultant no association might be due to smaller sample size in the present study.
\end{abstract}

Keywords: Cross-sectional Study, Indoor Air Pollution, Low Birth Weight, Pregnant Women, Solid fuel

\section{Introduction}

The use of solid fuels on open fires or in traditional stoves results in high level of indoor air pollution. Moreover, it can exceed its acceptable levels particularly in poorly ventilated dwellings. Women and young children are highly exposed to indoor solid fuel smokes as women spend most of her time cooking ${ }^{[1]}$. Indoor air pollution is responsible for over 1.6 million annual deaths and 2.7 percent of the global burden of disease (in Disability-Adjusted Life Years or DALYs). In developing countries, the major source of indoor air pollution is the burning of solid fuels i.e. biomass fuels (animal dung, wood, crop residues) and coal for heating and cooking. The estimation shows that, around $50 \%$ of the world's population is using solid biomass fuels for cooking and heating purpose. The exposure to indoor air pollution is excessively high among young children and women with exposure levels exceeding international health-based guidelines by more than or equal to 20 times ${ }^{[2]}$. Studies of urban air pollution and environmental tobacco smoke (ETS) revealed that combustion pollutants i.e. Carbon monoxide (CO) and small particles are associated with adverse pregnancy outcomes, still births and low birth weight. Smokes from solid fuels used in developing countries also contain combustion pollutants whose exposure is expected to have an impact on adverse pregnancy outcomes ${ }^{[3]}$. In India, the 1991 national census incorporated a question on the major household fuel used for the first time and revealed that around $95 \%$ of the rural population relied mainly on biomass fuels (dung, crop residues, and wood) and a small proportion using coal, which means about $97 \%$ of households, relied principally on these unprocessed solid fuels. Nationwide, some $81 \%$ of all households relied on solid unprocessed fuels, among these, around $3 \%$ used coal and $78 \%$ used biomass fuels. Similar findings were observed in a national survey of 89,000 households conducted in 1992. It is already known that such fuels are significantly more polluting than the liquid and gaseous fuels. Thus, the use of unprocessed fuels is considered as a heterogeneous proxy for the measurement of indoor air pollution ${ }^{[4]}$.

Burning of the solid fuels result in the production of carbon monoxide, organic compounds and other particulate matters ${ }^{[5,6]}$. It is believed that these organic compounds may probably get absorbed in maternal blood resulting to have direct effects on foetus ${ }^{[7]}$. The high level of exposure to carbon monoxide results in the formation of carbo-oxyhaemoglobin which reduces the oxygen carrying capacity of blood to different organs. Foetal blood has 10 times more affinity to bind the carbon-monoxide and also the elimination rate is slow as compared to mother. Thus a growing foetus might get deprived of oxygen resulting in the intra uterine growth retardation, still births, low birth weight or other adverse pregnancy outcomes ${ }^{[8]}$.

A study done on chronic low level maternal carbon monoxide exposure and fetal growth and development also revealed that exposure to carbon-monoxide has been associated with retarded fetal development and adverse pregnancy outcomes, including peri-natal mortality and reduced birth weight ${ }^{[9]}$. 
Smokes from biomass fuels contain pollutants such as polycyclic aromatic hydrocarbons, which may impair the mother's lung functions and may place mothers to develop acute or chronic lung diseases. The mother's weakening lung capacity also result in reducing oxygen delivery to the fetus. The maternal lung disease and impaired lung function have been associated with adverse pregnancy outcome i.e. still births, low birth weight, preterm delivery. ${ }^{[10]}$

Low birth weight is a most important determinant of mortality, morbidity and disability in infancy and childhood. It also has a long-term impact on health outcomes in adult life. Low birth weight also results in significant costs to the health sector and imposes a significant burden on society as a whole. The global prevalence of such births is slowly dropping however in developing countries; it is as high as $30 \%{ }^{[11]}$ The association between maternal exposures to household air pollution (HAP) and low birth weight have been described in number of studies, however only few studies have been conducted in India to establish the association. Hence this study was undertaken to identify if there was any association between exposure of pregnant women to indoor air pollution and birth weight of a newborn.

\section{Materials And Methods}

A community based cross-sectional study was conducted in Machche village of Kinaye primary health center for a period of one and half year. The data was collected from the pregnant mothers attending the antenatal checkup visits to the sub-centers during the study period. Universal Coverage was followed in this study. i.e. 254 (All registered pregnant mothers during January to November 2012). The pregnant mothers who were registered in the respective sub-centers \& were attending in the clinics for ANC checkups during the study period \& the mothers who gave consent were included in the study. On the other hand, pregnant women who were having a home delivery or having systemic illness(i.e. Gestational Diabetes, HIV Infection, Tuberculosis, Malaria, Genito-urinary infection, heart disease, renal disease, hypertensive disorder of pregnancy occurring after 20 weeks of gestation, having multiple gestation, having spontaneous abortion in current gestation, having a induced preterm delivery due to maternal \& fetal conditions) or pregnant mothers who chewed tobacco \& tobacco related products were excluded from the study. The exposure to passive smoking of participants was categorized into high (always/frequently around people smoking tobacco products indoor) or low (sometimes/rarely/never around people smoking tobacco products indoor. ${ }^{[12]}$

Participant's exposure to solid fuel was grouped into 3 categories using the method developed by Mishra et al i.e. participants using only solid fuels were considered at high risk, those using only non-solid fuels at low risk and those using mixture of solid and non solid fuels at medium risk ${ }^{[13]}$. Along with these data, information on socio-demographic profile was also collected. Pretested close ended questionnaire were administered for data collection. Data were collected from registers and interview as well. The data on exposure to indoor air pollution were collected on an interview schedule with the mothers. The pilot study was conducted on $10 \%$ of the pregnant mothers, before undertaking the major study. This was done to assess the feasibility of the study and applicability of the questionnaire. Minor changes were incorporated in the questionnaire after the pilot study. Participants in the pilot study were not included as subjects in the main study. Data were compiled, coded and analyzed in IBM SPSS software version 20. Appropriate statistical tests were applied wherever required.

\section{Results}

This study included the total of 254 mothers. Table 1 shows the distribution of mothers according to demographic variables. Among the total mother interviewed, majority of them were in the age group of 20-29 years. Rest of them were either more than 29 years of age i.e. $4.3 \%$ or less than 20 years i.e. $2.4 \%$. Majority of the mothers were Hindu i.e. $68.9 \%$ followed by Muslim i.e. $30 / 3 \%$ and very less i.e. $0.8 \%$ were Christians. Regarding educational status of the mother, majority of them had attained lower secondary education i.e. $33.1 \%$, followed by primary education i.e. $28 \%$ and secondary education i.e. $19.7 \%$. Very less were graduate being $2 \%$. Among total mothers, majority belonged to class III socioeconomic category i.e. $61 \%$ followed by class IV category i.e. $26.4 \%$ and rest of the others belonged to class I category. 
Table 1: Distribution of participants according to demographic variables

\begin{tabular}{lll}
\hline Demographic Variables & Frequency & Percent \\
\hline Age groups & & 2.4 \\
$\leq 19$ years & 6 & 93.3 \\
$20-29$ & 237 & 4.3 \\
$\geq 30$ & 11 & 100.0 \\
Total & 254 & 68.9 \\
Religion & & 30.3 \\
Hindu & 175 & .8 \\
Muslim & 77 & 100.0 \\
Christian & 2 & \\
Total & 254 & 9.4 \\
Educational status & & 28.0 \\
Illiterate & 24 & 19.7 \\
Primary & 71 & 7.9 \\
Secondary & 50 & 2.0 \\
PUC & 50 & 100.0 \\
Graduate & 20 & 12.6 \\
Total & 5 & 61.0 \\
Socio-Economic status & 254 & 26.4 \\
Class II & & 100.0 \\
Class III & & \\
Class IV & 32 & \\
Total & 155 & 67 \\
\hline
\end{tabular}

As seen in Fig 1, out of total mothers, only $2.8 \%$ delivered low birth weight babies and rest of them were normal birth weight.

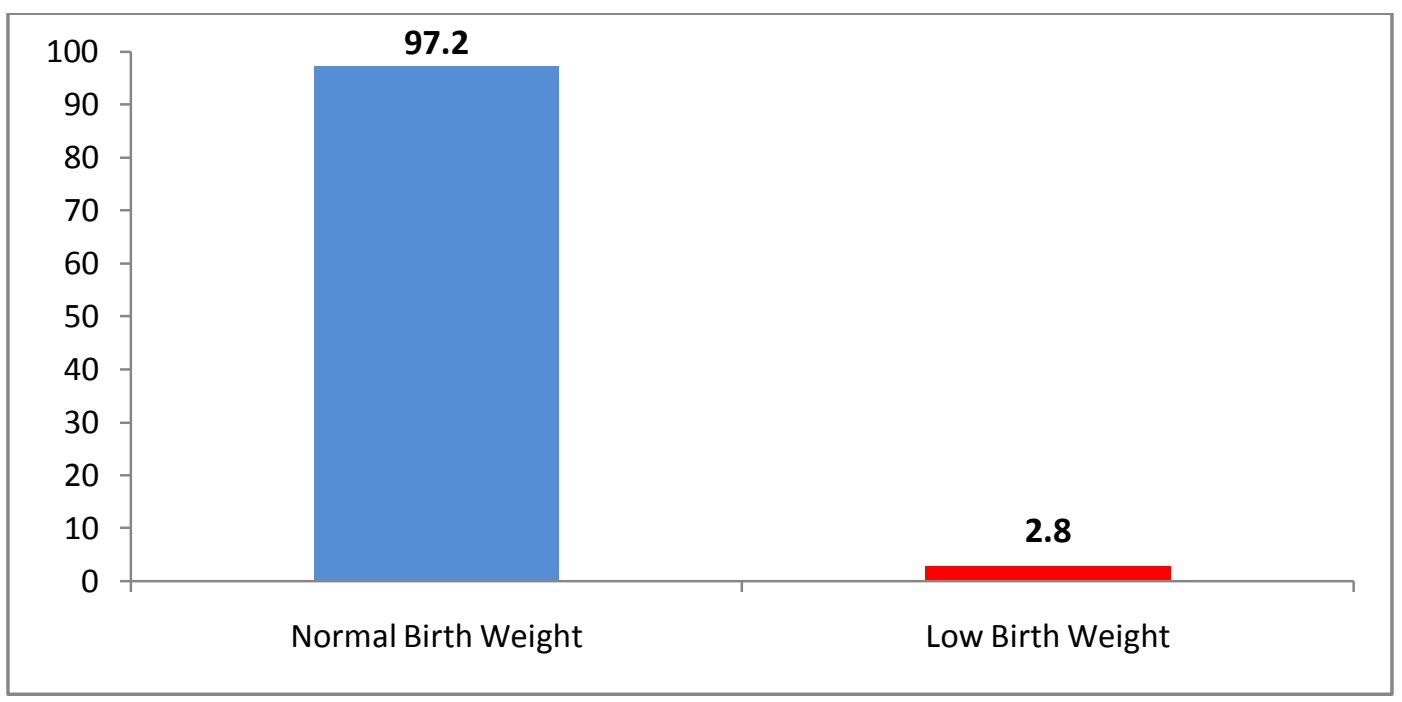

figure 1 percentage of low birth weight

Fig 2 shows the types of fuel used by mothers. Among the total mothers studied, majority of them were solid fuel users i.e. $35.4 \%$ (high risk groups), followed by both solid and non-solid fuel users (medium risk groups) i.e. $32.7 \%$ and remaining were non-solid fuel users (low risk groups). 


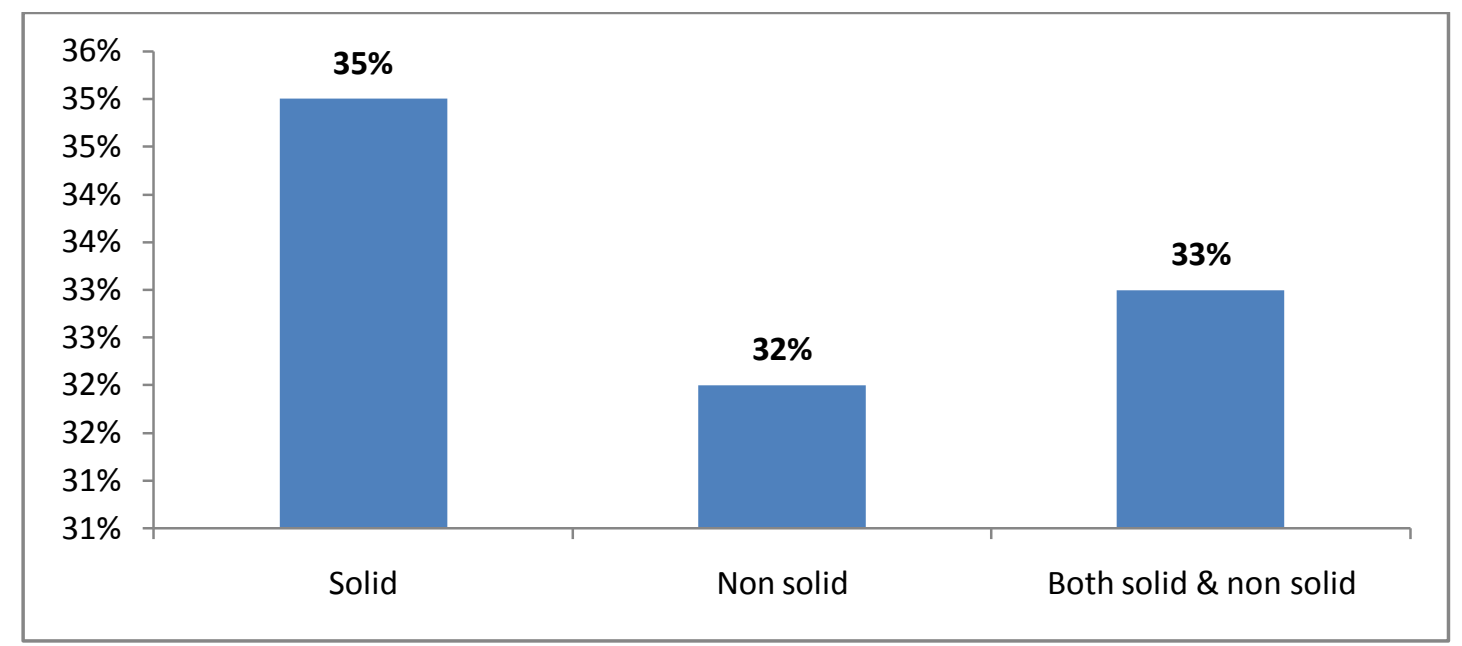

figure 2 types of fuel used

Similarly, Fig 3 describes the exposure of mothers to passive smoking where on assessing the risk of passive smoking, $30.7 \%$ were under high risk of passive smoking and rest of the others were under low risk of passive smoking.

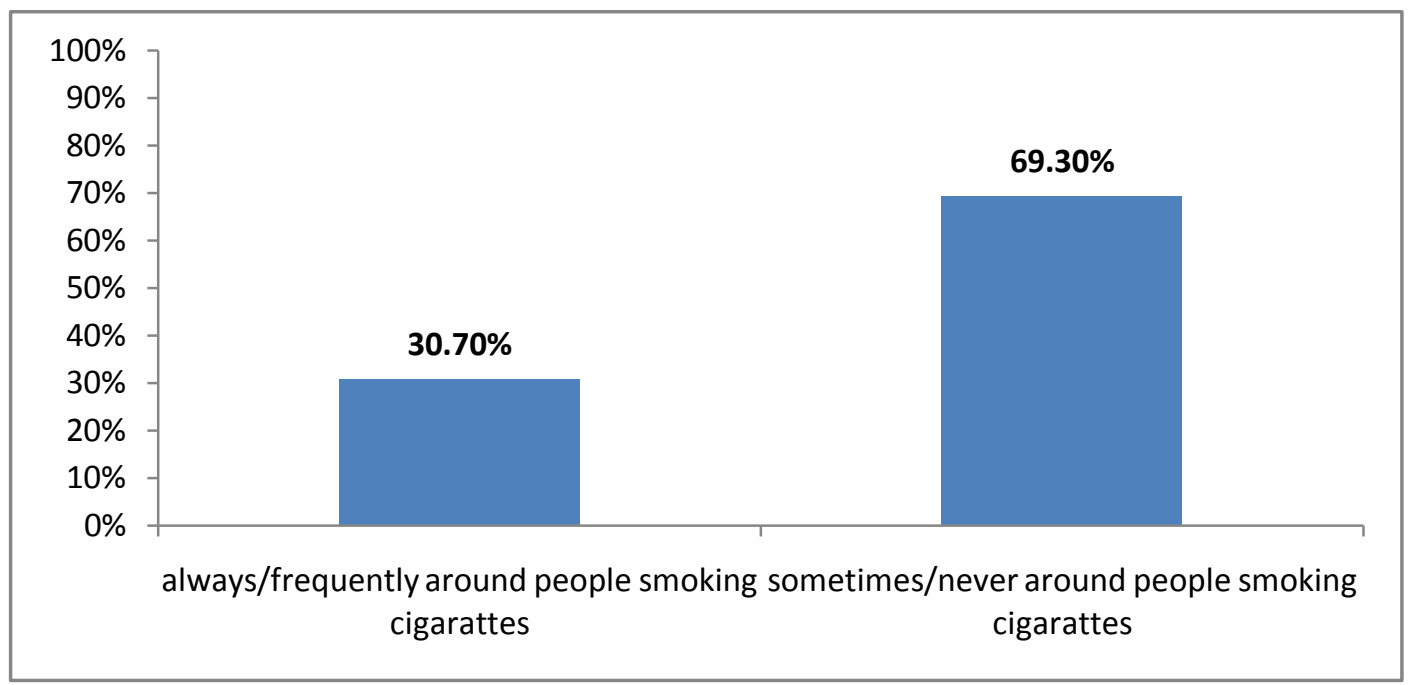

figure 3 exposure to passive smoking

Table 2 describes about the characteristics of kitchen where $91.3 \%$ were having separate room for kitchen. Only $76.28 \%$ had chimney in the kitchen. Majority of them were having indoor kitchen i.e. $88.2 \%$, only $11.4 \%$ had separate kitchen outside the house and very small percentage i.e. $0.4 \%$ had open air kitchen outside the house. Likewise, more than half of the house had no cross ventilation in their kitchen. Similarly, more than one third i.e. $40 \%$ of the mother were residing in semi-pukka house followed by pukka house i.e. $36.2 \%$ and $23.2 \%$ were residing in kachcha type of house

Table 2: Kitchen Characteristics

\begin{tabular}{lll}
\hline Kitchen Characteristics & Frequency & Percentage \\
\hline Separate room used as kitchen & & 91.3 \\
Yes & 232 & 8.7 \\
No & 22 & 100.0 \\
Total & 254 & \\
& & 23.2 \\
Presence of Chimney & & 76.8 \\
Yes & 59 & 100.0 \\
No & 195 & 254
\end{tabular}




$\begin{array}{lll}\begin{array}{l}\text { Location of Kitchen } \\ \text { Indoor kitchen } \\ \text { Separate kitchen outside the house }\end{array} & 224 & 88.2 \\ \begin{array}{l}\text { Open air kitchen outside the house } \\ \text { Total }\end{array} & 29 & 11.4 \\ & 1 & 4 \\ \text { Cross-ventilation in kitchen } & 254 & 100.0 \\ \text { No } & & \\ \text { Yes } & 132 & 52.0 \\ \text { Total } & 122 & 48.0 \\ & 254 & 100.0 \\ \text { Types of House } & & \\ \text { Kachcha } & 59 & 23.2 \\ \text { Pukka } & 92 & 36.2 \\ \text { Semi-Pukka } & 103 & 40.6 \\ \text { Total } & 254 & 100.0 \\ & & \end{array}$

Likewise, Table 3 reveals the association of educational status, types of fuel used and exposure to passive smoking with birth weight of the newborn. Educational status was found to be significantly associated with low birth weight i.e. $\mathrm{p}<0.05$. However the types of fuel used and exposure of the mother to passive smoking was not found to be significantly associated with low birth weight i.e. $p>0.05$. Table 4 reveals the association of socioeconomic status \& types of house with types of fuel used by mothers. Socio-economic status $\&$ types of house both were significantly associated with types of fuel used by mothers i.e. $p<0.05$. Table 5 shows the association between socio-economic status and exposure of mother to passive smoking, which was found to be significantly associated with one another i.e. $\mathrm{p}<0.05$

Table 3: Association of educational status, types of fuel used and exposure to passive smoking with birth weight of the newborn

\begin{tabular}{|c|c|c|c|c|c|}
\hline \multirow{2}{*}{\multicolumn{2}{|c|}{ Variables }} & \multicolumn{2}{|c|}{ Birth weight } & \multirow{2}{*}{ Total } & \multirow{2}{*}{$\begin{array}{c}\text { Chi-square, } \mathrm{df}, \mathrm{P}- \\
\text { value }\end{array}$} \\
\hline & & Low Birth Weight & Normal Birth Weight & & \\
\hline \multirow{2}{*}{$\begin{array}{l}\text { Educational } \\
\text { Status }\end{array}$} & Illiterate & 3 & 22 & 25 & \multirow{2}{*}{$8.842,1, .023$} \\
\hline & Literate & 4 & 225 & 229 & \\
\hline \multirow[b]{3}{*}{$\begin{array}{l}\text { Types of fuel } \\
\text { used }\end{array}$} & Solid & 3 & 87 & 90 & \multirow[b]{3}{*}{$1.040,2, .595$} \\
\hline & Non solid & 1 & 80 & 81 & \\
\hline & Both solid and non solid & 3 & 80 & 83 & \\
\hline \multirow{2}{*}{$\begin{array}{l}\text { Exposure to } \\
\text { passive smoking }\end{array}$} & High exposure & 3 & 75 & 78 & \multirow{2}{*}{$0.499,1,0.368$} \\
\hline & Low exposure & 4 & 172 & 176 & \\
\hline
\end{tabular}

Table 4: Association of socioeconomic status \& types of house with types of fuel used

\begin{tabular}{|c|c|c|c|c|c|c|}
\hline \multirow{2}{*}{\multicolumn{2}{|c|}{ Variables }} & \multicolumn{3}{|c|}{ Types of fuel used } & \multirow{3}{*}{$\begin{array}{c}\text { Total } \\
32 \\
\end{array}$} & \multirow{2}{*}{$\begin{array}{l}\text { Chi-square, } \mathrm{df}, \mathrm{P} \text { - } \\
\text { value }\end{array}$} \\
\hline & & Solid & Non solid & Both solid and non & & \\
\hline $\begin{array}{l}\text { Socio- } \\
\text { Economic } \\
\text { Status }\end{array}$ & Class II & 5 & 11 & 16 & & $7.487,2, .024$ \\
\hline \multirow{2}{*}{$\begin{array}{l}\text { Types of } \\
\text { House }\end{array}$} & Kachcha & 34 & 2 & 23 & 59 & \multirow[b]{2}{*}{$63.394,4,0.000$} \\
\hline & Pukka & 8 & 50 & 34 & 92 & \\
\hline
\end{tabular}

Table 5: Association between socioeconomic classification and exposure to passive smoking

\begin{tabular}{|l|c|c|c|c|}
\hline \multirow{2}{*}{$\begin{array}{l}\text { Socioeconomic } \\
\text { classification }\end{array}$} & High Risk & Low Risk & \multirow{2}{*}{ Total } & Chi-square, df, P-value \\
\cline { 2 - 5 } Class II & 5 & 27 & 155 & $16.141,2,0.000$ \\
\hline Class III & 62 & 93 & 67 \\
\hline Class IV & 11 & 56 & 254 \\
\hline
\end{tabular}




\section{Discussions}

Present study found $2.8 \%$ low birth weight among total deliveries in Machche Village, Kinaye. A retrospective study conducted at Kinaye primary health center found $8.3 \%$ prevalence of low birth weight in the year $2007-2010^{[14]}$. This difference in prevalence was observed probably due to small sample size in present study. Our study showed that the majority of the mothers were Hindu i.e. $68.9 \%$ followed by Muslims i.e. $30.3 \%$ and rest of them were Christians. A study done in Belgaum in the year $2010-2011$ showed that $86.7 \%$ of the mothers were Hindu and rests of the others were Muslims ${ }^{[14]}$. Another study done in Purliya district in West Bengal revealed that $88.8 \%$ of the mothers were Hindu and rests of them were Muslims similar to present findings ${ }^{[15]}$. Literate class constituted of $90.15 \%$ of the study population and rests of them were illiterate. Among literates, more than half i.e. 52.8 had attained secondary education, followed by primary education i.e. $28 \%$, PUC $7.9 \%$ and very few were graduates i.e. $2 \%$. Furthermore, educational status showed significant association with the birth weight of a newborn. Analogous to our findings, a study conducted in Goa medical college in the year 2006 showed that mothers with no education were likely to deliver a low birth weight infant [16]. Similarly, another study done in rural Guatemala also showed the same result where it was found that low birth weight was significantly associated with maternal literacy status ${ }^{[17]}$. Majority i.e. $61 \%$ of the mothers were in class III socio-economic scale followed by class IV i.e. $26.4 \%$ and remaining were in the class II scales. However no significant association was found between socio-economic status and low birth weight of newborn in present study. These results were consistent with that of Malaysian study where household income was not the risk factor for low birth weight ${ }^{[18]}$. However, Kramer in his meta-analysis found that poor socio economic condition was important risk factor of low birth weight ${ }^{[19]}$. A study conducted in Goa medical college also found that mothers belonging to lowest socioeconomic status were most likely to deliver low birth weight infants ${ }^{16}$. These inconsistencies in results were observed might be due to low sample size in present study. Among the total mothers studied, more than one third i.e. $35.4 \%$ of them were using solid fuels (high risk) for the purpose of cooking and heating. Similarly, $32.7 \%$ were using both solid and non solid fuels (medium risk) and remaining i.e. $31.9 \%$ were using non solid fuels (low risk) for cooking and heating purpose in their home. However, the study didn't find any association between the types of fuel used by mother and birth weight of a newborn. Contrary to our study, the findings from the study done in rural Wardha in 2008 found association with types of fuel used and low birth weight ${ }^{[20]}$. Another study done in rural Guatemala also showed that fuel types was significantly associated with reduced birth weight ${ }^{[17]}$. This difference observed might be due to relatively having a smaller sample size in the present study. With regard to the association between types of fuel used and demographic characteristics related to mothers, only socio-economic status was found to be significantly associated with types of fuel used. These findings are somehow comparable with the study done in southern India as they found significant association between types of fuel used and literacy status of the women and literacy status plays important contribution for socio-economic development ${ }^{[21]}$. On assessing the risk of passive smoking, more than two third i.e. $69.3 \%$ of the mother were at low risk groups and rest of them were at high risk. The results are however consistent with the study done in southern India where $19.9 \%$ of all respondents were exposed to high risk of passive smoking and remaining were in the low risk groups ${ }^{[21]}$. Association between passive smoking and low birth weight was not established in this study. Concurrently, study conducted among Malay women in Ota Bharu, Kelantan also revealed no evidence of association between passive smoking and low birth weight, supporting our study findings ${ }^{[22]}$. However a study done in Yale-New Haven Hospital established the association between passive smoking and low birth weight ${ }^{[23]}$. This difference might be due to small sample size in the present study. Furthermore, passive smoking was significantly associated with the socioeconomic status of the mother. These findings are also somehow comparable with the study done in southern India as they found second hand smoke exposure of pregnant women was associated with educational status of the women, education being contributor for socioeconomic development ${ }^{[21]}$. Present study explored that majority i.e. $91.3 \%$ of the mothers had separate room used as kitchen. Only $23.2 \%$ had chimney in the kitchen. About two third of the mothers had indoor kitchen, $11.4 \%$ of them had separate kitchen outside the house and less than $1 \%$ had open air kitchen outside the house. Similarly, more than one third i.e. $37 \%$ had cross-ventilation in their kitchen. More than half of the mothers reported that they spent 3 to 4 hours in the kitchen when stove is lit. On observing about the types of house, majority i.e. $40.6 \%$ of it was semi-pukka type followed by pukka type i.e. $36.2 \%$ and rest of the remaining were kachcha type houses. Among all of these characteristics of the kitchen, only types of house were found to be significantly associated with birth weight. Similarly, types of house were also significantly associated with the types of fuel used by mothers. These findings are consistent with the findings of study done in rural Guatemala where the types of fuel were significantly associated with the floor material of the house and birth weight of the newborn as well ${ }^{[17]}$. 


\section{Conclusion}

The percentage of low birth weight was $2.8 \%$ in Machche I \& II villages of Kinaye. Educational status was significantly associated with low birth weight. Types of fuel used and passive smoking were not found to be significantly associated with low birth weight. This resultant no association might be due to low sample size in the present study. Among the factors that were significantly associated with the types of fuel used were socioeconomic status of the mother and types of house where mothers reside. Similarly, socio-economic status was also significantly associated with exposure of mother to passive smoking. The relationship between exposure level of indoor air pollution and low birth weight needs to be further investigated by increasing the sample size and using more rigorous study designs including other rural areas. The use of direct measure of exposure status might yield more valid exposure status of the mothers to indoor smoke. However, the study was unable to explore all the other known co-variates of low birth weight. The data obtained of exposure to indoor air pollution and passive smoking was subjective i.e. based on respondents self report. Survey of limited sample of pregnant mothers might have imprecise the findings of the study.

\section{References}

[1]. World Health Organization. Indoor Air Pollution [internet] [cited 2011 December 31] Available from: http://www.who.int/indoorair/en

[2]. World Bank. Indoor Air Pollution (Environmental Health). [Internet] [cited 2012 March 2 ] Available from: http://web.worldbank.org/Wbsite/External/Topics/Environment/Extenvhea/0, ,contentMDK:21158391 menuPK:4367070 pagePK: 210058 piPK:210062 theSitePK:3662880,00.html

[3]. World Health Organization. Indoor Air Pollution from Solid Fuels and Risk of Low Birth Weight and Stillbirth. Report from a symposium held at the Annual Conference of the International Society for Environmental Epidemiology (ISEE), 2005 September

[4]. Smith K R. National Burden of Disease in India from Indoor Air Pollution. PNAS 2000; 97: 13286-13293

[5]. Bruce N, Perez-Padilla R, Albalak R. Indoor air pollution in developing countries: a major environmental and public health challenge for the new millennium. Bull World Health Organ. 2000; 78(9):1078-1092.

[6]. Naeher LP, Brauer M, Lipsett M, et al. Wood smoke health effects: a review. Inhal Toxicol. 2007;19(1):67-106.

[7]. Glinianaia SV, Rankin J, Bell R, et al. Particulate air pollution and fetal health: a systematic review of the epidemiologic evidence. Epidemiology. 2004; 15(1):36-45.

[8]. Ritz B, Yu F. The effect of ambient carbon monoxide on low birth weight among children born in Southern California between 1989 and 1993. Environ Health Perspect. 1999; 107(1):17-25.

[9]. Garvey DJ, Longo LD. 1978. Chronic low level maternal carbon monoxide exposure and fetal growth and development. Biol Reprod 19:8-14.

[10]. Edenborough FP, Stableforth DE, Webb AK, Mackenzie WE, Smith DL. 1995. Outcome of pregnancy in women with cystic fibrosis. Thorax 50:170-174

[11]. World Health Organization. Feto-maternal nutrition and low birth weight [internet]. [Cited: 2013 Jan 16]. Available from: http://www.who.int/nutrition/topics/feto_maternal/en/).

[12]. Kelly PJ, Goudar SS, Chakraborty $\bar{H}$, Moore J, Derman R, Kodkany B, Bellad M, Naik VA, Angolkar M, Bloch M. Pregnant women and children's exposure to tobacco and solid fuel smoke in southwestern India. J Matern Fetal Neonatal Med. $2011 ; 24(7): 973-7$

[13]. Mishra V, Dai X. Smith R K, Mika L. Maternal Exposure to Biomass Smoke and Reduced Birth Weight in Zimbabwe. Ann Epidemiol 2004; 14:740-747

[14]. Metgud C. S., Naik V. A. \& Mallapur M. D. Consanguinity and Pregnancy Outcome among Rural Pregnant Women of Belgaum District. Community Med, 2012;3(4): 681-4.(22)

[15]. Biswas R., Dasgupta A., Sinha, R. N. \& Chaudhuri, R. N. An epidemiological study of low birth weight newborns in the district of Puruliya, West Bengal. Indian Journal of Public Health 2008; 52(2):65-71.

[16]. Roy S., Motghare D., Ferreira A., Vaz F., Kulkarni M. Maternal Determinants of Low Birth Weight at a Tertiary Care Hospital. The Journal of Family Welfare. 2009; 55(1): 79-83

[17]. Boy E, Bruce N and Delgado H. Birth Weight and Exposure to Kitchen Wood Smoke during Pregnancy in Rural Guatemala. Environmental Health Perspectives 2002 January; 110(1): 109-114

[18]. Boo NY, Lim SM, Koh KT and Lau KF. Risk factors associated with low birth weight infants in the Malaysian population. Med J Malaysia. 2008; 63(4):306-10

[19]. Kramer MS. Determinants of low birth weight: methodological assessment and meta-analysis. Bull World Health Organ. 1987; 65(5):663-737. Available from: http://www.ncbi.nlm.nih.gov/pubmed/3322602. (Accessed on 25.06.2011 at 19:00hrs IST)

[20]. Boratne A. V., Gupta S. S., Datta S. S., Mehendale A. M., Garg B. S. Indian Journal of Maternal and Child Health. Indian Journal of Maternal and Child Health. 2012. 14(2):1-9

[21]. Kelly PJ, Goudar SS, Chakraborty H, Moore J, Derman R, Kodkany B, Bellad M, Naik VA, Angolkar M, Bloch M. Pregnant women and children's exposure to tobacco and solid fuel smoke in southwestern India. J Matern Fetal Neonatal Med. 2011;24(7):973-7

[22]. Norkhajizah S et al. Higher incidenc of low birth weight infants among Malay women with periodontitis in Ota Bharu, Kelantan. Jurnal Kesihatan Masyarakat lsu Khas 2004:10-12.

[23]. Martin T. R., Bracken, M. B. Association of low birth weight with passive smoke exposure in pregnancy. American Journal of Epidemiology. 1986; 124(4): 633-64 